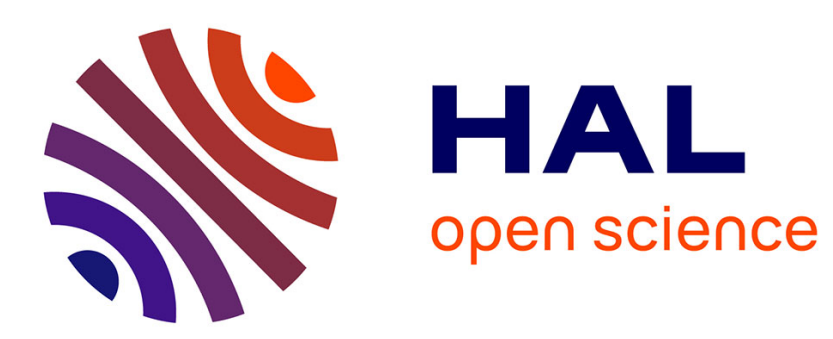

\title{
A propositional representation model of anatomical and functional brain data
}

\author{
Pablo Maturana, Bénédicte Batrancourt
}

\section{To cite this version:}

Pablo Maturana, Bénédicte Batrancourt. A propositional representation model of anatomical and functional brain data. Cinquième conférence plénière française de Neurosciences Computationnelles, "Neurocomp'10", Aug 2010, Lyon, France. hal-00553437

\section{HAL Id: hal-00553437 \\ https://hal.science/hal-00553437}

Submitted on 16 Mar 2011

HAL is a multi-disciplinary open access archive for the deposit and dissemination of scientific research documents, whether they are published or not. The documents may come from teaching and research institutions in France or abroad, or from public or private research centers.
L'archive ouverte pluridisciplinaire HAL, est destinée au dépôt et à la diffusion de documents scientifiques de niveau recherche, publiés ou non, émanant des établissements d'enseignement et de recherche français ou étrangers, des laboratoires publics ou privés. 


\title{
A PROPOSITIONAL REPRESENTATION MODEL OF ANATOMICAL AND FUNCTIONAL BRAIN DATA
}

\author{
Pablo MATURANA, Bénédicte BATRANCOURT \\ CRICM, UMR_S975, INSERM/CNRS, Pierre et Marie Curie Univ. \\ Hôpital Pitié-Salpêtrière, Paris \\ France \\ pablo.maturana_quijada@upmc.fr, benedicte.batrancourt@upmc.fr
}

\begin{abstract}
A great number of natural systems can be represented by networks. Recent developments in the domain of networks have been transfered to the neurosciences. For example, the graph model has been used in neuroimaging research as a methodological tool to examine brain network organization, topology and complex dynamics, as well as as a framework to test the structure-function hypothesis.

In the current work we propose a network of anatomical brain data and neuropsychological assessments linked to the functions explored in these assessments, offering a platform to visualize, in a single graph-theoretic framework, anatomo-functional and neuropsychological information.

We apply this framework to a database belonging to the Center of Cognitive Anatomy (CAC), which contains neuropsychological data of patients presenting sequels of focal brain damage.
\end{abstract}

\section{KEY WORDS}

Brain, Atlas, Graph, Neuropsychology, Ontology and Modelling.

\section{Introduction}

The principle of anatomo-functional correlation in neuropsychology proposes to establish a link between: i) imagery data reflecting a characterization at the structural, brain anatomical level, ii) information measuring the cognitive performance of a patient or the intensity of a psychological or behavioral trait, obtained with different instruments such as neuropsychological tests and neurological or behavioral scales. Generally, anatomo-functional correlations are established on the basis of statistical treatment. We propose an alternative conceptual framework to establish anatomo-functional correlations:

- The definition of a propositional representation model of the anatomical and functional brain data implementation based on the graph and network theory [1], [2].

- The treatment of the embedded graph data as a way to explore and to analize information.

Complex network analysis has its origins in the mathematical study of networks, known as graph theory. However, unlike classical graph theory, this analysis mainly deals with real-life networks that are large and complex neither uniformly random nor ordered [3].

We propose this new framework of computational tools to be applied to neuropsychological data. It would incite the emergence of rules linking the anatomical and functional data and it would provide stable "anatomofunctional laws" which should be independent of all inter-individual brain variability.

\section{Methods}

Graph theory is usually considered as the most appropriate framework for the mathematical treatment of complex networks. In general, a complex network is represented by nodes and edges where the nodes correspond to the elements of the system and the edges to the interactions between them [4].

To study brain networks in relation to the anatomofunctional information, we defined a general structure in two levels:

- A basic layer associated to a physical and structural level, which supports the embedded graph anatomical and functional data.

- An upper layer associated to an application level, which offers tools to explore and treat the graphs, making possible the emergence of new associations between anatomical and functional data.

For the basic layer we designed a tripartite graph consisting of three distinct sets of nodes. One set corresponds to the information provided by the thesaurus proposed in BrainInfo [5]. This hierarchical thesaurus has about 1580 cerebral entities (nodes of the graph) classified as a tree. The other two sets of nodes correspond to the functional and neuropsychological assessment data obtained from the NeuroLOG project. This data includes the neuropsychological instruments (tests and scales) used for the assessments, the variables measured by these instruments, the associated scores obtained by patients, the domains and cognitive functions or processes explored by the different instruments.

GraphML [6] and GEXF [7] (Graph Exchange XML Format) have been used to represent the data. Both are extensions of XML language and are data formats specific for graphs. GEFX, as compared to GraphML, has the advantage that allows the visualization of attributes ( $\mathrm{x}, \mathrm{y}, \mathrm{z}$, color and size) and the design of dynamic graphs. This characteristics are useful to visualize the network in Talairach coordinates.

In the upper layer, we use two tools: one to visualize and another one to analyze the graphs. For the 
visualization we used the software Gephi [8]. Gephi is an interactive visualization and exploration platform for all kind of networks and complex systems, dynamic and hierachical graphs. It is a collaborative open-source project developed in Java and it uses OpenGL as the visualization engine. To analyze the graphs we used the bioinformatic Matlab toolbox. This toolbox allows to apply graph theory algorithms to sparse matrices. A graph can be represented as a sparse matrix, where nonzero entries represent the edges and the values of these entries represent the weight associated to the edge (cost, distance, lenght or capacity).

\section{Results}

\subsection{Anatomical graph}

The anatomical information (thesaurus from BrainInfo), see Fig. 1, consisted of about 1,580 cerebral entities (nodes of the graph) classified as a tree. The hierarchical relations of the thesaurus constitute the edges of the graph (1,619 edges). These relations are of the type "is a part of", i.e. they are essentially descriptive and correspond to the grouping of subgroups of elements according to the principal types of organisation of the central nervous system: cortical, neighbourhood, histological, chemical and, more recently, an organisation on the basis of the white matter connectivity.

In a future version, we will add new relations such as:

- Relation of type "functional", linking entities engaged in the same cognitive task

- Relation of type "connectivity", linking entities connected by white fibber bundles.

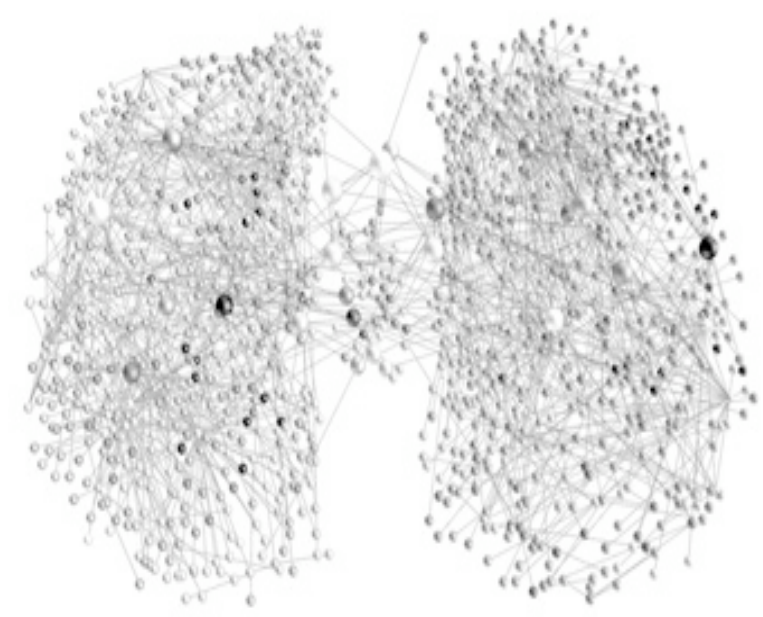

Fig. 1. The anatomical graph has been created from the data available in BrainInfo (1,580 nodes and 1,619 edges of the type: "is a part of") and visualized with Gephi. The left and rights graph partitions represent the left and right cerebral hemispheres.

\subsection{Neuropsychological assessments and functional graph}

In the framework of the NeuroLOG project (ANR), we proposed a core ontology of instruments OntoNeuroLOG [9], that may facilitate the representation and sharing of results of explorations with neurological and neuropsychological instruments. This ontology modelizes in the form of artifacts, the information involved in an assessment score. It takes into account the structure, generally hierarchical and composition, of such instruments and makes it possible to relate their different variables to brain functions or abilities measured when the tests were taken.

These neuropsychological assessments have assigned scores that result from a particular patient's cognitive performance (e.g. calculation performance) or trait intensity (e.g. pessimistic thoughts intensity) in relation to the instrument variable. For instance a Score may correspond to the following propositions: "During one MMS Calculation assessment, Patient X's Calculation performance is equal to 4 as measured by such MMS Calculation variable".

The functional graph (see Fig. 2) is aligned on the ontology OntoNeuroLOG. We have described a first set of 50 cognitive functions (e.g. memory, language) and 16 neuropsychological instruments (e.g. the MiniMental State - MMS). The functional relationships are:

- Relation of type "sub-instrument" linking a particular test with its correspond sub-tests

- Relation of type "explore" linking a particular test with a cognitive function or domain.

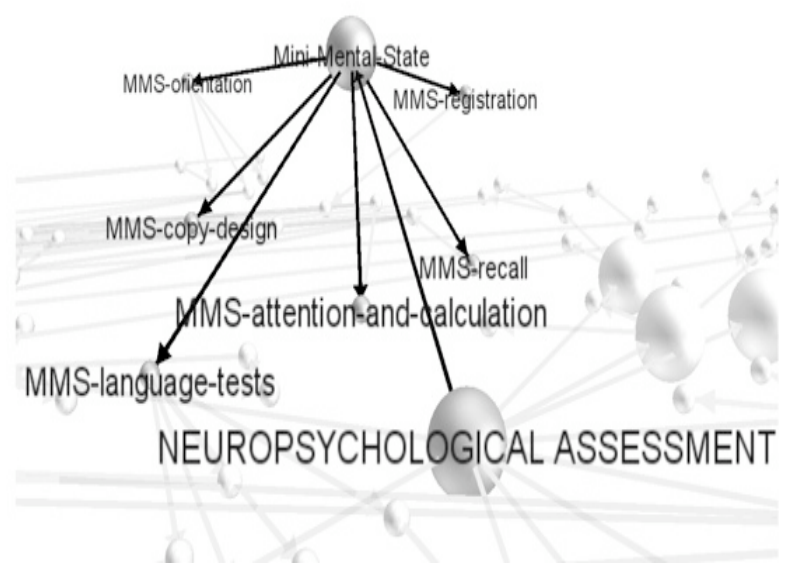

Fig. 2. A part of the functional graph has been aligned on the ontology OntoNeuroLOG and visualized with Gephi. The graph partition represents the Mini-Mental State test (MMS) and its sub-tests like MMS-recall or MMS-copy-design. 


\subsection{Patient data}

Once the anatomical and functional graphs have been developed, we proceeded to add neuropsychological data of 10 patients with brain damage see Fig. 3 and Fig. 4. For this purpose, we used the database belonging to the Center of Cognitive Anatomy (CAC), located at the Pitié-Salpêtrière Hospital, Paris. The $\mathrm{CAC}$ was set up for the collection and processing of neuronatomical and neuropsychological data of patients presenting sequels of focal brain damage.

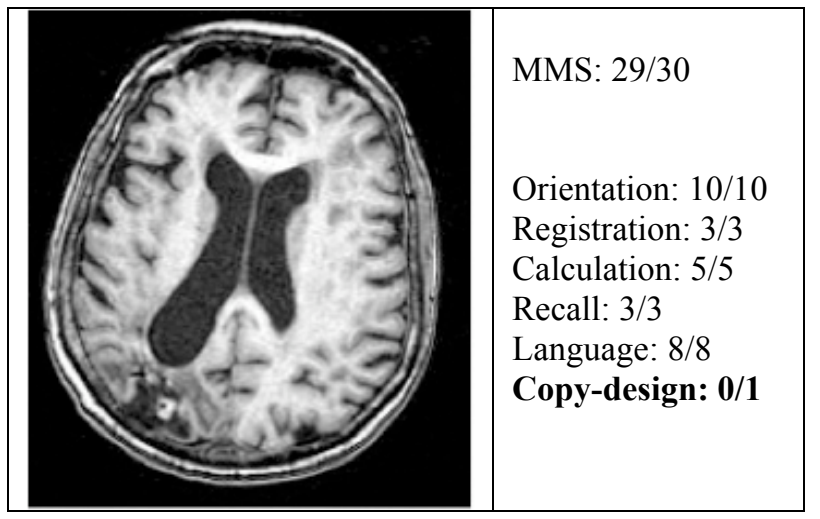

Fig. 3. A CAC patient case (PCA120). The lesion is located in the right parietal cortex and includes the angular gyrus (AnGr). The neuropsychological assessment revelead that he/she presented a deficit according to the score $(0 / 1)$ obtained on the MMSCopy-design sub-test.

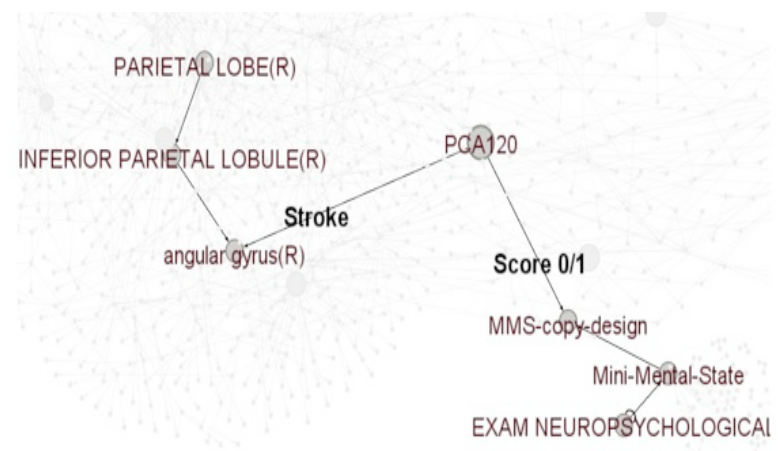

Fig. 4. The corresponding anatomo-functional graph of the CAC patient (PCA120). On the left, we present the functional graph partition, which corresponds to the MMS test and its sub-tests. On the right, the anatomical graph partition corresponding to the right parietal lobe is shown. The node PCA120 corresponds to the patient case. The edge labelled Score $0 / 1$ relies the node $P C A 120$ to the MMS-Copy-design sub-test node, showing the relevant neuropsychological information. The edge labelled Stroke relies the node PCA120 to the right Angular Gyrus (AnGr) showing the localisation of the lesion.

\section{Conclusion and future perspectives}

The first layer of our framework offers a trustable architecture: an embedded graph of anatomical and functional data, implemented with the languages GraphML and GEXF
As an expansion of this first step, we will:

- Emphasize the anatomical graph with the currently anatomo-functional knowledge and more specially concerning the brain white matter connectivity.

- Develop neurocomputational tools (with Gephi and Matlab) to explore and treat the graphs in order to reveal other posible anatomo-functional relations.

\section{Acknowledgements}

This work is supported by IFRAD (International Fund Raising for Alzheimer's disease).

\section{References}

[1] B. Batrancourt, S. Bonnevay, M. Lamure, R. Levy \& B. Dubois, An Anatomofunctional Brain Knowledge. Proc. 5th ISIF Information Fusion 2002, Annapolis, MD, Washington DC Area, USA, 2002, 1269-1276.

[2] B. Batrancourt , R. Lévy, S. Lehericy, D. Hasboun, Y. Samson, I. Lavallée, M. Lamure \& B. Dubois, Création d'une base de données sur le cerveau pour une synthèse anatomo-fonctionnelle, C. R. Biologies (C) Académie des sciences / Éditions scientifiques et médicales, Elsevier SAS, 325, 2002, 1-17.

[3] M. Rubinov, O. Sporns, Complex network measures of brain connectivity: Uses and interpretations, NeuroImage, 2009.

[4] Boccaletti, S., Latora, V., Moreno, Y., Chavez, M., Hwang, D.-U., Complex networks: structure and dynamics. Phys. Rep 2006. 424, 175-308.

[5] BrainInfo (2007), Neuroscience Division, National Primate Research Center, University of Washington, http://www.braininfo.org

[6] GraphML@ 2001-2007 GraphML Working Group, http://graphml.graphdrawing.org/

[7]GEXF 2009 GEXF Working group, http://gexf.net/format/

[8]Gephi.org (c) All Rights Reserved 2008-2010, http://gephi.org/

[9] B. Batrancourt, M. Dojat, B. Gibaud, \& G. Kassel, A core ontology of instruments used for neurological, behavioral and cognitive assessments. Proc. 6th FOIS International Conference on formal Ontology in Information Systems 2010, Toronto, Ca, 2010. 